\title{
Multi-state Empirical Valence Bond study of temperature and confinement effects on proton transfer in water inside hydrophobic nanochannels
}

\author{
Amani Tahat, Jordi Martí
}

April 12, 2016

\begin{abstract}
Microscopic characteristics of an aqueous excess proton in a wide range of thermodynamic states, from low density amorphous ices (down to $100 \mathrm{~K}$ ) to high temperature liquids under the critical point (up to $600 \mathrm{~K}$ ), placed inside hydrophobic graphene slabs at the nanometric scale (with interplate distances between 3.1 and 0.7 $\mathrm{nm}$ wide) have been analyzed by means of molecular dynamics simulations. Waterproton and carbon-proton forces were modelled with a Multi-state Empirical Valence Bond method. Densities between 0.07 and $0.02 \AA^{-3}$ have been considered. As a general trend, we observed a competition between effects of confinement and temperature on structure and dynamical properties of the lone proton. Confinement has strong influence on the local structure of the proton, whereas the main effect of temperature on proton properties is observed on its dynamics, with significant variation of proton transfer rates, proton diffusion coefficients and characteristic frequencies of vibrational motions. Proton transfer is an activated process with energy barriers between 1 and $10 \mathrm{~kJ} / \mathrm{mol}$ for both proton transfer and diffusion, depending of the temperature range considered and also on the interplate distance. Arrhenius-like behavior of the transfer rates and of proton diffusion are clearly observed for states above $100 \mathrm{~K}$. Spectral densities of proton species indicated that in all states Zundel-like and Eigen-like complexes survive at some extent.
\end{abstract}

Keywords: $\quad$ Proton transfer, graphene slab, temperature and confinement effects, Empirical Valence Bond, Molecular Dynamics.

*Department of Physics, Technical University of Catalonia-Barcelona Tech, B4-B5 Northern Campus UPC. Jordi Girona, 1-3. 08034 Barcelona. Catalonia. Spain. 


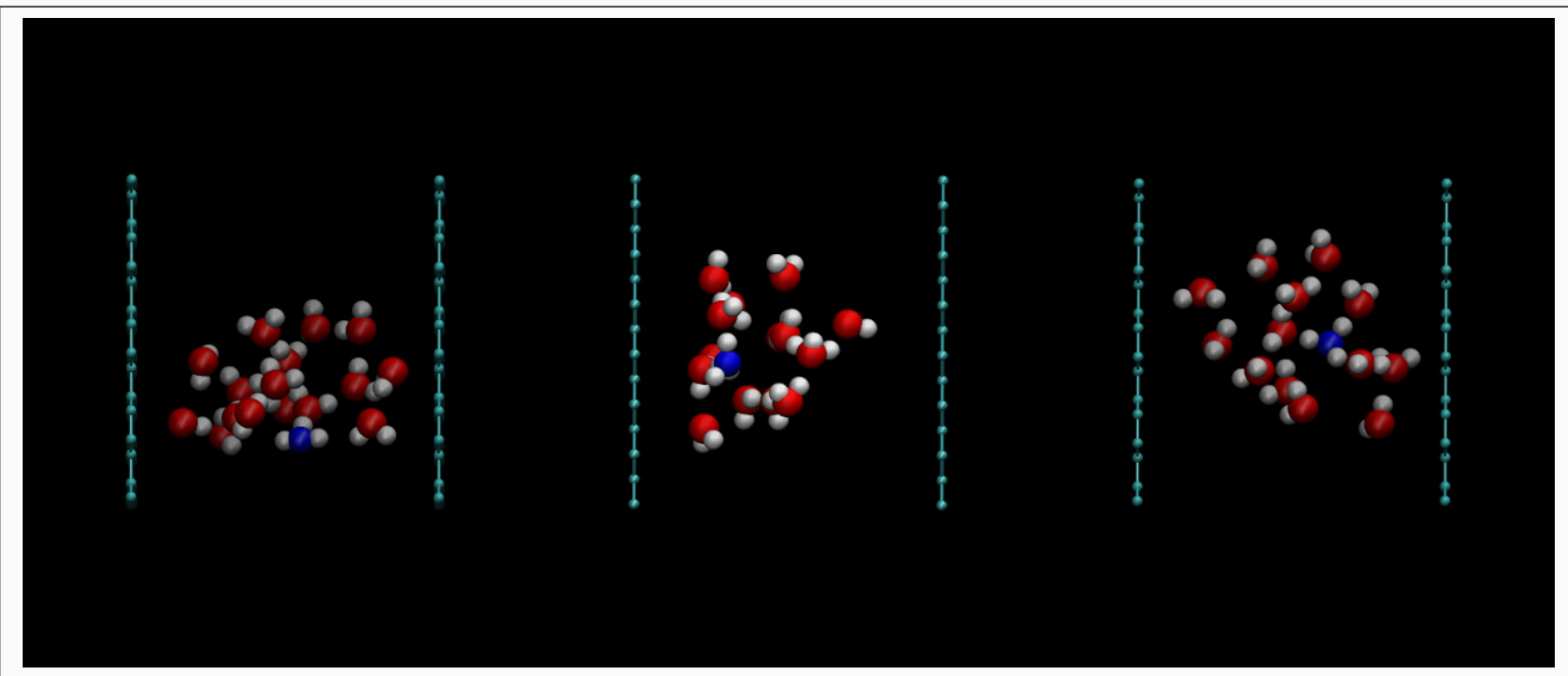

(75 words.) Snapshots of local configurations around the pivot water carrying the excess proton confined inside the graphene slab $1.5 \mathrm{~nm}$ wide, at different thermodynamic states (left to right): $\mathrm{T}=100,300$ and $500 \mathrm{~K}$. 


\section{INTRODUCTION}

Proton transfer (PT) in aqueous media is a widespread fundamental process in nature and technology, playing an important role in fields such as water autoionization, ${ }^{1}$ molecular mechanisms related to the human immunodeficiency-1 protease virus, ${ }^{2}$ nicotine activation in water media, ${ }^{3}$ molecular reactions in aerosols ${ }^{4}$ or in energy conversion processes like photosynthesis or in cellular respiration, ${ }^{5}$ to mention just a few. In bulk water and bulk ice there are solid indications that protons are not transported by ordinary diffusion, but by special conduction mechanisms such as the transfer mechanism initially suggested by Von Grotthuss long time $\operatorname{ago}^{6-8}$ and consisting of the continuous jump of the proton through the wires created by hydrogen bonds (HB), where each oxygen atom simultaneously passes and receives a single hydrogen atom. After recent findings from both experimental and theoretical studies, a modern image of the lone proton has arisen. ${ }^{9-26}$. From this image, there is a general consensus that for bulk water proton dynamics is directly associated with dynamics of the HB network of water.

Nevertheless, aqueous proton transport processes occurring in complex, strongly confined environments, are not yet fully understood. In many cases proton conduction takes place in confined volumes, such as in the case of PT along biomembranes, ${ }^{24}$ in small embedded water nanopools within proteins and peptides, ${ }^{27}$ for excess protons near alumina surfaces ${ }^{28}$

or through water channels like those of nafion membranes in hydrogen fuel cells. ${ }^{29-31}$ The understanding of proton conduction in confined geometries is thus essential to comprehend and ultimately control a wide variety of biological and technological systems of fundamental and practical interest. Despite the large scientific and industrial relevance of PT, the mechanisms of proton conduction are still a largely unexplored area of research. The main reason for this missed knowledge is essentially the limited number of experimental techniques sufficiently sensitive to probe proton conductivity in confined spaces, and also the lack of accurate predictions coming from theory and simulation. Up to date, it has been observed that when confined in restricted geometries, microscopical properties of the proton also suffer drastic changes from those at bulk states.

At the surroundings of the lone proton we should expect significant differences with local 
coordination shells of pure liquids, ices and vapors. However, since the calculation of the phase diagram of the water model employed in this work out of the scope of this paper, we will consider a series of equally distributed thermodynamical states from low density amorphous (LDA) ices to dense liquids at variable densities and temperatures and explore the influence of such factors on the structural and dynamical characteristics of PT. We should point out that given the complexity of the phase diagram of water ${ }^{32,33}$, studies of microscopical characteristics of aqueous lone protons are usually reported only for selected groups of thermodynamical states corresponding to restricted regions of the diagram. For instance, multi-state empirical valence bond (MS-EVB) calculations of PT in water confined in carbon nanotubes ${ }^{34}$ confirmed early results $^{35}$ revealing that PT rates inside the tubes are about one order of magnitude faster than in bulk. Recently, a proposed new classical model with explicit proton transfer by Wolf and Groenhof ${ }^{36}$ has provided a way to model PT in a very efficient computational procedure using classical force fields, but still capturing the key aspects of the phenomenon. The most accurate but computational expensive simulations by ab-initio molecular dynamics (MD) of water inside nanochannels ${ }^{37,38}$ revealed significantly different mobilities for hydroxyl $\mathrm{OH}^{-}$and hydronium $\mathrm{H}_{3} \mathrm{O}^{+}$ions, depending on the size of the tubes and the degree of functionalization of the tube walls. Very recent quantum-classical simulations by Bankura and Chandra ${ }^{39}$ indicate that when water is strongly confined in two-dimensional environments, the lone proton is likely to be solvated as the Eigen cation and that PT rates are significantly lower than in the quasi-one-dimensional case.

It should be pointed out that spectroscopical data have been very valuable for the interpretation of the PT phenomenon. So, for instance, characteristic signatures of Zundel and Eigen species have been observed in photoevaporation of weakly bound argon by means of photofragmentation mass spectroscopy and compared to ab-initio data at MP2 level $^{40,41}$, with overall good agreement. On the other hand, infrared spectroscopy of protonated benzene-water nanoclusters ${ }^{42}$ suggested local ordering of the first water shell around the proton. Summarizing, despite that there is plenty of information on microscopic properties of lone protons in water, the influence of a variety of factors on them, from variations in thermal energy to hydrophobicity effects, is still far from well understood. Based on a previous work on computer simulations of excess protons confined in narrow nanometric 
channels at room conditions ${ }^{43}$, we will report in the present paper a thorough analysis of the influence of both temperature and confinement on systems such as protons in LDA ices and in hot water, in order to get further information of local structures and dynamics of the lone proton.

\section{METHODOLOGY}

In this study we employed a MS-EVB approach to model the quantum nature of proton, observed through its delocalization, together with MD techniques which allowed us to monitor the trajectory of all particles in time. Although this combined methodology has been widely used to study chemical reactivity in solution ${ }^{11,14,17,19,26,43-51}$, we will describe the most important details of the methodology below.

First, it is important to note that quantum effects may be described with several methods, essentially depending on the amount of quantum degrees of freedom present in the system under study. However, full quantum calculations such those performed with as ab-initio or path integral methods are extremely expensive for systems containing, say, more than 32 molecules (see for instance discussion in Ref. ${ }^{23}$ ). In our case, the system consists of a single quantum-like particle (the proton) in a classical bath of 125 solvating waters, where methods such as MS-EVB are very appropriate. MS-EVB methods assume that a Born-Oppenheimer potential energy surface $\epsilon_{0}(\{\mathbf{R}\})$ is driving the dynamics of the nuclei with coordinates $\{\mathbf{R}\}$ and it can be obtained from the lowest instantaneous eigenvalue of the EVB Hamiltonian:

$$
\hat{\mathrm{H}}_{\mathrm{EVB}}(\{\mathbf{R}\})=\left|\phi_{i}\right\rangle h^{i j}(\{\mathbf{R}\})\left\langle\phi_{j}\right|
$$

where we have considered (as it will be in most of the forthcoming formulas) the criterion

of summation over repeated indexes. The Hamiltonian $\hat{\mathrm{H}}_{\mathrm{EVB}}(\{\mathbf{R}\})$ is represented in terms of a basis set $\left\{\left|\phi_{i}\right\rangle\right\}$ of diabatic (localized) VB states. In the present setup, the diabatic states are associated to configurations with the $\mathrm{H}^{+}$attached to a given oxygen. The ground-state $\left|\psi_{0}\right\rangle$ of $\hat{\mathrm{H}}_{\mathrm{EVB}}$ satisfies:

$$
\hat{\mathrm{H}}_{\mathrm{EVB}}\left|\psi_{0}\right\rangle=\epsilon_{0}(\{\mathbf{R}\})\left|\psi_{0}\right\rangle
$$


It can be expanded as a linear combination of diabatic states as:

$$
\left|\psi_{0}\right\rangle=\sum_{i} c_{i}\left|\phi_{i}\right\rangle
$$

leading to the following potential energy surface:

$$
\epsilon_{0}\{\mathbf{R}\}=c_{i} c_{j} h^{i j}(\{\mathbf{R}\})
$$

Finally, dynamics of the nuclei of mass $M_{k}$ is governed by the following Newton's equation of motion:

$$
M_{k} \frac{\mathrm{d}^{2} \mathbf{R}_{k}}{\mathrm{~d} t^{2}}=-c_{i} c_{j} \nabla_{\mathbf{R}_{k}} h^{i j}(\{\mathbf{R}\})
$$

In the framework of EVB methods, off-diagonal elements of the Hamiltonian matrix $h_{i j}$ can be casted out in terms of nuclear coordinates, achieving an excellent agreement with results from full quantum calculations. It should be pointed out that the parameterization employed in the present work ${ }^{46,49,50}$ has been successfully tested for a wide variety of environments of the aqueous proton. Diagonal elements $h_{i i}$ included contributions from stretching and bending vibrations within the tagged $\mathrm{H}_{3} \mathrm{O}^{+}$and also inside the rest of water molecules, which have been modeled using the parameters of the flexible TIP3P force field given by Dang and Pettitt ${ }^{52}$. The intramolecular interactions consisted in a Morse term for the stretching forces and a harmonic term for bending, whereas they are modeled as purely harmonic interactions (for both stretching and bending parts) for the atom-atom intramolecular forces in water. The diagonal elements also include intermolecular interactions such as those between hydronium-solvent and solvent-solvent.

Off-diagonal elements $h_{i j}$ introduce the coupling between diabatic states $i$ and $j$ and have been modeled including interatomic contributions within a particular water pair spanned by states $\left|\phi_{i}\right\rangle$ and $\left|\phi_{j}\right\rangle$ plus Coulomb interactions between the dimer and the rest of molecules of the solvent. A complete list of parameters is provided in Ref. ${ }^{49}$. Finally, oxygen-carbon and hydrogen-carbon forces were modeled by Lennard-Jones forces with the same parameterization employed in previous works. ${ }^{53}$ So, using Lorentz-Berthelot mixing rules, we got $\sigma_{O C}=3.28 \stackrel{\circ}{A} \sigma_{H C}=2.81 \stackrel{\AA}{A} \varepsilon_{O C}=0.389 \mathrm{~kJ} / \mathrm{mol} \varepsilon_{H C}=0.129 \mathrm{~kJ} / \mathrm{mol}$. 
The process of construction of the EVB Hamiltonian follows a series of steps: First, we identify the water molecule closest to the lone proton; this particular water forms the initial pivot $\mathrm{H}_{3} \mathrm{O}^{+}$and the first diabatic state. From this, the rest of the diabatic states are chosen in a tree-like construction through the hydrogen-bond network. As a criterion for a hydrogen bond to be formed, we considered the maximum oxygen acceptor-proton donor distance up to $2.8 \AA$ and we imposed a minimum threshold value of the $\mathrm{H}-\mathrm{O}-\mathrm{O}$ angle of $30^{\circ}$. All molecules lying in up to the third solvation shell of the pivot species showing HB connections were included in the construction of the $L \times L$ EVB Hamiltonian matrix, which must be properly diagonalized. $L$ is usually of the order of $\sim 10$ units. At each time step, PT corresponds to the re-assignment of the pivot oxygen label to the instantaneous state having the largest $c_{i}^{2}$ coefficient (Eq.1). From this state, the list of participating VB states was reconstructed each time step using the procedure described above. After the EVB matrix was formed, ground-state eigenvectors and Hellmann-Feynman forces were given by:

$$
\mathbf{F}_{k}=-c_{i} c_{j} \frac{\partial \hat{H}_{E V B}^{i j}(\mathbf{x})}{\partial \mathbf{x}_{k}} .
$$

All simulation experiments corresponded to microcanonical runs at the average temperatures of $T=100,200,300,400,500$ and $600 \pm 20 \mathrm{~K}$. Fluctuations in the total energy were of about $1 \%$ of the averaged value for all simulated systems. In particular, at $300 \mathrm{~K}$ and for the interplate distance of $1.5 \mathrm{~nm}$, the average in total energy was of $\langle E\rangle=175.2 \pm 1.4$ $\mathrm{kcal} / \mathrm{mol}$, with a contribution from the excess proton of $25.8 \pm 0.2 \mathrm{kcal} / \mathrm{mol}$ and from the bath of $149.1 \pm 1.3 \mathrm{kcal} / \mathrm{mol}$. The average in temperature was of $\langle T\rangle=298.3 \pm 1.6 \mathrm{~K}$. No drift of total energy was observed in all cases.

In order to keep the HB network formed up to some extent (and, eventually to have the possibility of PT episodes at all states) we increased the density of the system from $0.02 \AA^{-3}$ (3.1 nm wide slab) to $0.07 \AA^{-3}(0.7 \mathrm{~nm}$ wide slab). This means that the simulations were performed with 125 water molecules in all cases into a slab of width ranging from $d=3.1$ to $0.7 \mathrm{~nm}$. The boxlenght along $\mathrm{X}$ and Y coordinates was of $15.6 \stackrel{\circ}{A}$ in all cases, whereas the simulation boxlenght along the Z-coordinate was enlarged five times the value of the $\mathrm{X}(\mathrm{Y})$ direction, in order to avoid interactions between image graphene sheets ${ }^{54}$.

According to the phase diagram of the rigid TIP3P model ${ }^{33}$ the state at $300 \mathrm{~K}$ correspond 
to liquid water and those of 200 and $100 \mathrm{~K}$ to low density amorphous ices (LDA) ices. However, these assignments are only approximate, since (1) our model includes flexibility of the molecular bonds and (2) the pressure in our system fluctuated within a range up to $15 \%$ of the mean value. Temperature variations were always small (within 3\%). States over $300 \mathrm{~K}$ (400 to $600 \mathrm{~K}$ ) have not been evaluated for the present model, but we believe that at the pressures considered in this work, all of them will correspond to liquid or very dense vapor states. Finally, our time step was set to $\Delta t=0.5$ fs for all simulations. We considered equilibration periods of approximately $20 \mathrm{ps}$, followed by trajectories of more than $0.25 \mathrm{~ns}$, used to obtain meaningful statistical properties. Long ranged forces (Coulomb interactions)

were handled by Ewald sum techniques ${ }^{55}$, using a uniform neutralizing background charge in all cases. This is satisfactory enough for a system including a small charge as the lone proton, whereas in extended systems such as proteins of membranes in water the use of a counterion would be in order ${ }^{56}$.

\section{RESULTS and DISCUSSION}

\section{Local structure of the hydrated proton}

In a similar fashion as it happens when a solvated ion in water produces a large anomaly in the tetrahedral structure of the bulk liquid, the presence of an excess proton also creates a disruption in its local hydrogen-bond structure. When a PT event occurs, the structure around the lone proton changes dramatically and three relevant complexes may arise: the proton attached to a single water (single hydronium, $\mathrm{H}_{3} \mathrm{O}^{+}$), the so-called Zundel dimer $\left(\mathrm{H}_{5} \mathrm{O}_{2}\right)^{+57}$ and the three-coordinated hydronium $\left(\mathrm{H}_{9} \mathrm{O}_{4}\right)^{+}$known as the Eigen complex ${ }^{58}$. It is commonly accepted that PT is the result of the continuous interconversion of the three above mentioned structures, with percentages of each depending on the thermodynamic conditions of the system. In most cases, continuous interconversions between the Zundel and Eigen complexes generate a hybrid $\left(\mathrm{H}_{9} \mathrm{O}_{4}\right)^{+} /\left(\mathrm{H}_{5} \mathrm{O}_{2}\right)^{+}$structure ${ }^{59,60}$.

Each transfer of the lone proton between two water molecules will involve changes in pivot oxygen-water oxygen $\left(\mathrm{O}^{*}-\mathrm{O}\right)$ and pivot oxygen-water hydrogen $\left(\mathrm{O}^{*}-\mathrm{H}\right)$ distances and changes 
of the local hydrogen connectivity pattern between the complex at its closest solvation shells. We expect that both temperature and confinement will have observable effects on the local proton structure. As usual we can analyze solvation structures by means of local pivot oxygen-water $(\mathrm{O}, \mathrm{H})$ density fields given by:

$$
\rho_{\mathrm{O}^{*} \alpha}(r)=\frac{1}{4 \pi r^{2}}\left\langle\sum_{i} \delta\left(\left|\mathbf{r}_{\mathrm{O}^{*}}-\mathbf{r}_{i}^{\alpha}\right|-r\right)\right\rangle,
$$

where $\mathbf{r}_{\mathrm{O}^{*}}$ is the coordinate of the instantaneous pivot oxygen and $\mathbf{r}_{i}^{\alpha}$ is the coordinate of site $(\alpha=\mathrm{O}, \mathrm{H})$ in the $i$-th solvent molecule.

$\rho_{\mathrm{O}^{*} O}(r)$ are represented in the left side and $\rho_{\mathrm{O}^{*} H}(r)$ in the right side panels of Fig.1. From the former we can distinguish a marked first solvation shell centered around $r=2.4 \AA$ for all interplate distances with a slight tendency to increase localization as $d$ decreases. The location of this maximum is in good agreement with the findings of Bankura and Chandra ${ }^{39}$. This fact indicates that the proton is able to promote a remarkable extent of solvent clustering in its close vicinity, regardless of the temperature considered, from ice-like ambients to high sub-critical temperatures, in a close fashion to what is seen at ambient conditions for the unconstrained case (see Ref. ${ }^{26}$ ). A second solvation shell located at $\sim r=4.25 \AA$ at ambient conditions for the widest graphene channels $(d=3.1,1.5 \mathrm{~nm})$ can be also observed for the low temperatures but it quickly disappears above $300 \mathrm{~K}$. This is consistent with the picture of the lone proton loosing most of its hydrogen-bond connections to waters potentially participating of a second coordination shell. In the particular case of $d=0.7 \mathrm{~nm}$, the distance between the two plates is so short that, in average, there is only room available for two water layers. Such strong confinement effect of the graphene plates produces some reduction in size of the water second coordination shell at all temperatures, eventually shifting towards lower distances, centered around $\sim r=3.8-4 \AA$. This indicates that the local cluster around hydronium tends to become smaller as confinement becomes more important. This larger extent of water localization is similar to the case of cubic ice ${ }^{12,13}$ and of unconstrained amorphous ice $^{26}$.

Analysis of the oxygen pivot-hydrogen water density profiles is based on $\rho_{\mathrm{O}^{*} H}(r)$ at the right side of Fig.1. Here, in all cases, we found main peaks located around $r=3 \AA$, in a close fashion as it was recently reported from ab-initio and quantum-classical simulations ${ }^{39}$. As 
a general trend the height of this peak diminishes as temperature increases. At the lowest temperature such peak includes exclusively the six hydrogen atoms contained in the first solvation shell of the hydronium. As temperature increases the width of this band becomes larger (the first minimum shifts by $0.25 \AA$ ) and the averaged number of hydrogen atoms indicated by coordination numbers raises to $\sim 9-10$. At $100 \mathrm{~K}$ the structure of water hydrogens around the pivot oxygen enhances, suggesting a tendency of the system to evolve towards a solid-like configuration for all considered interplate distances. Finally, we didn't find any evidence of pivot acceptor hydrogen bonding of the type $\mathrm{O}-\mathrm{H} \cdots \mathrm{O}$, formed by means of the lone sp3 orbital of the acceptor oxygen, in the usual way of most of studies of confined water.

To provide a visual perspective of the local structure of the protonated water, we report selected snapshots of molecules around the lone proton having the largest weighting coefficients $c_{i}$ (of the order of $15-25$ molecules) for the intermediate distance $d=1.5 \mathrm{~nm}$ at temperatures of 100, 300 and $500 \mathrm{~K}$. From this picture we can observe that the proton together with its local environment (first water shell) mostly consist of a three coordinated Eigen cation, which keeps the proton separated from the graphene layers. We couldn't find any configuration where the hydronium was in direct contact with the surface. Conversely, for the closest $d=0.7 \mathrm{~nm}$ case, some configurations of the proton show it in direct interaction with the carbon walls, whereas for the $d=3.1 \mathrm{~nm}$ case, the local structure is more populated and the proton tends to be around the central part of the system (see Fig.1 of Ref. $\left.^{43}\right)$.

\section{Proton transfer dynamics}

Perhaps the most direct way to analyze dynamics' of proton transfer is by direct inspection of how the label of the pivot oxygen changes in time. We monitored pivot oxygen's label during 50 ps time intervals, as shown in Fig.3. There we compared the six temperatures considered in the present study for the three interplate distances of $3.1,1.5$ and $0.7 \mathrm{~nm}$ together with corresponding results for the bulk, unconstrained systems. The proton hopping patterns can be regarded as a sequence of episodes in which the proton is attached to one 
particular water during short to long time intervals, combined with other intervals in which the proton resonates between two given valence bond states. The latter is usually called proton rattling, with a "special" (resonant) bond ${ }^{48}$. Isolated single spikes reveal attempts of aborted transitions. From Fig.3 the frequency of proton transfer episodes is directly seen, by simply counting the number of transitions recorded: At $T=300 \mathrm{~K}$ (panels at the first column from the left), about $\sim 20$ water molecules retain the pivot label during time intervals of $\sim$ $0.5 \mathrm{ps}$ or longer, which roughly corresponds to a transfer time of $0.4 \mathrm{ps}^{-1}$. This is similar to the values that we can extract at higher temperatures. However, at lower temperatures the number of transitions decreases dramatically, and only $\sim 5$ are seen at $200 \mathrm{~K}$ and 2 at $100 \mathrm{~K}$. When the system is placed inside the graphene slab, the number of PTs clearly diminishes and is virtually zero for constrained states at $T<300$. At room temperature, PT in all cases are seen at frequencies much lower than for the unconstrained counterparts. So, for instance, the number of transitions for $d=3.1 \mathrm{~nm}$ at $600 \mathrm{~K}$ is around 7 but at the $d=0.7 \mathrm{~nm}$ case is only of $4-5$. Here it should be pointed out that the predicted rate of PT at ambient conditions (order of $0.5 \mathrm{ps}^{-1}$ ) is a factor $\sim 8$ larger than values reported from NMR experiments ${ }^{61-63}$. This is a known deficiency of the semi-classical model adopted here, although explicit incorporation of quantum fluctuations in the model of transferring proton produces a better agreement with experiments ${ }^{46}$.

\section{Proton transfer rates}

The rough picture of PT dynamics described from Fig.3 cannot provide a quantitative estimation of PT rates. However, we may improve the calculations using time correlation functions. In order to do this, we need to ensure that PTs are sufficiently frequent to collect statistics. This happened in all cases for the full length of our simulations (250 ps). The general form of equilibrium time correlation functions for the population relaxation of different reactant species is as follows ${ }^{50,64}$ :

$$
C(t)=\frac{\left\langle\delta h_{i}(t) \cdot \delta h_{i}(0)\right\rangle}{\left\langle\left(\delta h_{i}\right)^{2}\right\rangle}
$$

where $\delta h_{i}(t)=h_{i}(t)-\left\langle h_{i}\right\rangle$ describes the instantaneous fluctuation of the population of 
$i$-th reactant away from its equilibrium value. The characteristic function $h_{i}(t)$ is 1 if the tagged reactant species is present in the system at time $t$ and 0 otherwise. As it is usual in EVB-MD simulations of PT in water, population relaxations of the pivot label are the most appropriate stochastic functions to compute. With such choice, it has been observed that time correlation functions of $h_{i}(t)$ will likely show three different time scales characterized by different times: (1) a resonant time $\tau_{r s n}$ in the subpicosecond domain, corresponding to a rapid exchange of the pivot label along the "special" bond described above, i.e. associated with the spikes observed in the history of the pivot labels of Fig.3; (2) an intermediate time $\tau_{\text {prs }}$ characterizing the lifetime of the resonance episodes and (3) the residence time $\tau_{r s d}$ of the proton when attached to one particular pivot water. This time should be equivalent to the integrated relaxation time defined in Ref. ${ }^{39}$. Our results for the population relaxation of the pivot label are shown in Fig.4. The presence of several relaxation times is clear from the shapes of the correlation functions, showing at least one fast decay at short times (up to $0.5 \mathrm{ps}$ ) and a slow decay starting around 1.5 ps. Using Onsager's regression hypothesis ${ }^{65}$, proton transfer rates $k_{p}$ can be extracted from the long time slope of $C(t)$ :

$$
k_{p}=\lim _{t \rightarrow \infty}-\frac{\mathrm{d} \ln C(t)}{\mathrm{d} t} .
$$

Correspondingly, average mean residence time of the proton in a pivot water are estimated from $\tau_{r s d}=k_{p}^{-1}$. Full results for all the thermodynamical states considered in this work are reported in Table 1. We observe a general trend for all cases: relaxation times of $C(t)$ are systematically reduced when the system is heated up from LDA ice states (100 and $200 \mathrm{~K})$ to sub-critical high temperature states $(500$ and $600 \mathrm{~K})$. This means that proton transfer rates increase and, equivalently residence times $\tau_{r s d}$ decrease. The comparison between the three interplate separations reported in the present work indicates a clear trend of PT slowdown when the distance becomes smaller. Further, the numerical values obtained for the widest distance of $3.1 \mathrm{~nm}$ are quite similar to those found for the unconstrained systems (see Table 1) and close to the values reported by Bankura and Chandra ${ }^{39}$ for a single $H^{+}$in a water monolayer. However, when separations are smaller (1.5 and, especially, $0.7 \mathrm{~nm}$ ) PT rates decrease dramatically. When considering other simulation works, our data are in good overall agreement with findings from Day et al. ${ }^{50}$, who obtained a value for the proton transfer rate 
of $0.3 \mathrm{ps}^{-1}$ at room temperature $(300 \mathrm{~K})$, for an EVB model slightly different of the one used in the present work. For cubic ice, it was reported ${ }^{12,13}$ that the ratio between PT rates of liquid and ice phases is around 2, fact essentially attributed to the larger extent of localization of $O-O^{*}$ pairs, which could be at the basis of the PT mechanism in ices. In order to further investigate the temperature dependence of PT rates, we represented $\log k_{p}$ versus $T^{-1}$ in Fig.5 and explored the tendency to Arrhenius-like dependence and obtain the eventual activation energies $E_{k}$ for PT. We assumed the following dependence of the proton transfer rate with temperature

$$
k_{p} \sim A e^{-\frac{E_{k}}{k_{B} T}}
$$

where $A$ is a proportional factor and $k_{B}$ is Boltzmann's constant. From the calculation of the slopes of the straight lines shown in the Arrhenius plots of Fig.5, we get the series of values of $E_{k}$ reported in Table 2. A fit to the full set has been considered (continuous lines) and, since PT rates at $100 \mathrm{~K}$ seem to show a different trend, a fit to temperatures between 200 and $600 \mathrm{~K}$ (dashed lines) has also been taken. In the latter case, regression coefficients indicate a larger extent of Arrhenius-like behavior. Available experiments by Moon et al. ${ }^{66,67}$, by Kim et al. ${ }^{68}$ and by Luz and Meiboom ${ }^{69}$ obtained values of the order of $10 \mathrm{~kJ} / \mathrm{mol}$ for the activation energy of PT at the surface of polycrystalline ice films (135 $\mathrm{K}$ ), when the PT is mediated by hydroxyl ions and for PT in pure water, respectively, using methods such as reactive ion scattering and proton magnetic relaxation measurements. In our case, the values reported in Table 2 are between 2 and $12 \mathrm{~kJ} / \mathrm{mol}$. When fitting the full set of data, activation energies are around $4 \mathrm{~kJ} / \mathrm{mol}$ for unconstrained water and between 2 and $5 \mathrm{~kJ} / \mathrm{mol}$ for the confined setups, with the highest activation energy corresponding to the strongest confinement, i.e. when the separation between graphene plates is the shortest. If the fit is considered only for temperatures between 200 and $600 \mathrm{~K}$, values of activation energies rise but showing the same trend described above.

In summary, confinement does not alter significantly the energy barrier for activation of PT calculated for the free unconstrained liquid, with the exception of $d=0.7 \mathrm{~nm}$, where PT becomes a process requiring energy much bigger than at interplate distances of 3.1 and $1.5 \mathrm{~nm}$. This suggests that the slowdown of PT at low temperatures and severe confinement 
is mainly due to the lack of thermal energy and to the high degree of localization of the proton. In this way, the relationship between the likeliness of PT and the oxygen-oxygen distances of solvating water molecules was proposed some time ago by Marx ${ }^{23}$. The main idea was that at short distances, say for the Zundel dimer, there is a minimum in the external potential of the proton when located along the $\mathrm{O}-\mathrm{O}$ axis at equal distances of the two oxygens. Conversely, when the proton is closer to one of them the potential shows a maximum. However, Marx suggested that the correct picture should consider a twodimensional proton external potential depending of two variables: $R_{O-O}$ and the proton displacement coordinate $\mathbf{r}_{p}$. It should be noted that the need of multidimensional reaction coordinates for PT in water (autoionization process) had been already suggested by Geissler et al. ${ }^{1}$. From our results, the connection between O-O distances and activation energies for PT can be observed from Fig.1, where we found that states at stronger confinement $(d=0.7$ $\mathrm{nm}$ ) produced $\mathrm{O}-\mathrm{O}$ distances shorter than those of milder confinement $(d=3.1,1.5 \mathrm{~nm})$, in direct connection with the findings described above of overall highest activation energies for the strongest confined setups.

\section{Diffusion of the lone proton}

The calculation of diffusion coefficients in small systems can produce finite size effects. This is especially true in pure, bulk systems with isotropy along all three spacial directions (see

for instance the work by Yeh and Hummer ${ }^{70}$ ). When an interface is present, a suitable way to obtain the diffusion coefficient of a particle is by means of the projection of normal and parallel components of the atomic velocities ${ }^{71}$. However, in the present case the proton it has been shown (section ) that it tends to stay not in direct contact with the interface. Finally we should point out that proton's diffusion benefits from Grotthuss mechanism, operating through the HB network. Consequently, the relevant factor for diffusion is the existence of HBs formed between the proton and the surrounding water molecules. This fact has been corroborated in all cases, even in the closest separation of $0.7 \mathrm{~nm}$.

So, in the present MD+MS-EVB simulations the self-diffusion coefficients of aqueous protons $D_{p}$ were obtained from long time slopes of mean square displacements of the proton coordinate $\mathbf{r}_{p}$, following Einstein's relationship: 


$$
D_{p}=\frac{1}{6} \lim _{t \rightarrow \infty} \frac{\mathrm{d}}{\mathrm{d} t}\left\langle\left|\mathbf{r}_{p}(t)-\mathbf{r}_{p}(0)\right|^{2}\right\rangle,
$$

where the proton coordinate was defined as a weighted sum of the coordinates of the $L$ pivot molecules ${ }^{50} \mathbf{r}_{p v t}^{i}$ :

$$
\mathbf{r}_{p}=\sum_{i}^{L} c_{i}^{2} \mathbf{r}_{p v t}^{i}
$$

In the present case, as it was considered before ${ }^{43}$, the systems under study allow some mobility of the proton species along the Z-direction so that we kept the factor $\frac{1}{6}$ in the formulas instead of the factor $\frac{1}{4}$ considered in pure $2 \mathrm{D}$ simulations ${ }^{39}$. As our reference, we know that diffusion coefficients of aqueous protons at ambient conditions are about four times those of neat water: The experimental value of $0.93 \AA^{2} / \mathrm{ps}^{72}$ for a lone proton in water at $298.15 \mathrm{~K}$ and at the density of $1 \mathrm{gcm}^{-3}$ should be compared with the experimental diffusion coefficient of bulk liquid water of $0.23 \AA^{2} / \mathrm{ps}^{73}$. In the present case, the ratio of water and proton diffusion coefficients at $298 \mathrm{~K}$ has been of: 5 (unconstrained case); 6.9 $(d=3.1 \mathrm{~nm}) ; 26(d=1.5 \mathrm{~nm})$ and $11(d=0.7 \mathrm{~nm})$. This corroborates the well-known fact that MS-EVB models show a clear tendency to overestimate proton diffusion compared to that of water. The large difference between them is mainly due to the existence of the Grotthuss translocation mechanism, ${ }^{6}$ plus the hydrodynamic Stokes mass diffusion. In our case, mean square displacements of the proton are shown in Fig.6 and the corresponding diffusion coefficients are reported in Table 1. In general, the values of $D_{p}$ reported in the present work are of the same order of magnitude as those from Ref. ${ }^{39}$ for a single water layer inside graphene plates.

From mean square displacements two general trends are observed: (1) at the widest graphene-graphene separation of $d=3.1 \mathrm{~nm}$, proton diffusion coefficient is significantly larger than at the shortest $d$ (note that the same scale has been adopted for all cases); (2) within each $d$ diffusion decreases monotonically with temperature. So the general fact is that proton mobility is limited by confinement and enhanced by temperature. When numbers are considered, our first observation concerns the reliability of the model and methods considered here: we get a value of $D_{p}=0.94 \AA^{2} / \mathrm{ps}$ for the reference setup of room temperature for the 
unconstrained system, in excellent agreement with the experimental value reported above. This value remains unaltered for $d=3.1 \mathrm{~nm}$ but decreases roughly by a factor 4 in the cases of $d=1.5$ and $0.7 \mathrm{~nm}$. When we cool down the system and reach the range of LDA states (100 and $200 \mathrm{~K})$, obviously $D_{p}$ decreases drastically down, up to values of one $(d=1.5 \mathrm{~nm})$ to two $(d=0.7 \mathrm{~nm})$ orders of magnitude smaller. Here we deal with qualitative changes already observed experimentally in hexagonal ice networks ${ }^{74}$, where the proton turns down from a highly mobile solute at ambient conditions into a much slower particle at lower temperatures. As it has been pointed out, transport properties of the proton are similar to those of a small cation such as $\mathrm{Li}^{+}$, whose diffusion coefficient is about $0.1 \AA^{2} / \mathrm{ps}$ at $298 \mathrm{~K}^{19}$. In the range of high temperatures (400 to $600 \mathrm{~K}$ ), the behavior tends to remain more stable, with changes up to a factor 3 in the most extremal case (again for $d=0.7 \mathrm{~nm}$ ) where diffusion rises from 0.27 at $300 \mathrm{~K}$ to $0.93 \AA^{2} / \mathrm{ps}$ at $600 \mathrm{~K}$. So we obtain a more important influence of temperature over confinement in the proton diffusion. In contrast to the findings reported by Petersen et al. about the mobility of a proton near to the vapor interface ${ }^{75}$, in our simulations the single proton does not show the same tendency. We believe that this fact is probably due to the combination of two effects: (A) in the work by Petersen et al. ${ }^{75}$ the hydrophobic interface was the vacuum, whereas in our case we deal with a graphene sheet. Both surfaces are clearly of different nature, with the hydrophobicity of vacuum being milder than that of carbon surfaces, since in the former case there were no repulsive forces acting on the proton and in the latter, the interaction proton-carbon is strongly repulsive at short distances; (B) in our simulation experiments, we deal with important pressure gradients along Z-direction due to the presence of the graphene sheets, what produces a reduction of the proton diffusion and, presumably, a tendency to move in trajectories on planes parallel to the interfaces, as it was observed to happen in bulk water ${ }^{76}$.

In the same fashion as for PT rates, we can consider proton diffusion as an activated process (see Fig.7) and evaluate linear fits of $\ln D_{p}^{+}$as a function of $T^{-1}$, including or excluding the case of $100 \mathrm{~K}$, which turns out again to be the most controversial (see Table 2). In this case the overall fits (continuous lines) render activation energies slightly smaller than those from fits up to $200 \mathrm{~K}$ (dashed lines). However, the values are always in the range 2.5 to 6 $\mathrm{kJ} / \mathrm{mol}$. These values are in general very far from experimental findings reporting activation 
energies of water self-diffusion, between 14 and $70 \mathrm{~kJ} / \mathrm{mol}$ for water at ice surfaces and in bulk, respectively ${ }^{67}$. We observed that the Arrhenius-like behavior is more marked for the cases excluding the $100 \mathrm{~K}$ set.

\section{Spectral densities of the proton. Vibrational modes}

The widest employed experimental tools for the study of the microscopic vibrations of water are Raman and infrared spectroscopy. Infrared spectra are obtained by absorption coefficients $\alpha(\omega)$ or by means of the imaginary part of the dielectric constant, $\varepsilon^{\prime \prime}(\omega)^{77}$. These are quantum properties that can be naturally incorporated to the MD-EVB framework by using the absorption lineshape function $I(\omega)$, i.e. the Fourier transform of the time derivative of the dipole moment $\dot{\mu}(t)^{48,78}$. However, these functions may include oscillations that eventually reduce the quality of the spectral densities obtained from MD. Another approach that has been proved useful is the computation of velocity autocorrelations of the lone proton

$$
C_{p}(t)=<\mathbf{v}_{p}(0) \cdot \mathbf{v}_{p}(t)>
$$

where proton velocities $\mathbf{v}_{p}(t)$ can be obtained directly from the time derivative of its position $\mathbf{r}_{p}$ :

$$
\mathbf{v}_{p}(t)=\frac{\mathrm{d} \mathbf{r}_{p}(t)}{\mathrm{d} t}
$$

Using Eqs.(10) and (11) and taking Fourier transforms we can obtain vibrational densities of states $S_{p}(\omega)^{49}$ :

$$
S_{p}(\omega)=\int_{0}^{\infty} \mathrm{d} t C_{p}(t) e^{i \omega t} .
$$

With this procedure, we have computed $S_{p}(\omega)$ for a variety of thermodynamic states considered along the present work. For the sake of clarity, not all states have been included in Fig.8, only those showing significant features. All $C_{p}(t)$ have been computed up to 0.5 ps, time long enough to capture all relevant proton vibrations and safely shorter than the proton residence time (see Table 1), in order to make sure that the proton is attached to a given pivot oxygen during the whole period of calculation of $C_{p}(t)$. As the general fact, we 
will report the relevant modes of vibration of the proton inside a hydronium $\mathrm{H}_{3} \mathrm{O}^{+}$complex, either being part of a Zundel or an Eigen species. As usual, we included in our results a comparison to the corresponding $S_{p}(\omega)$ obtained from supplementary simulations ${ }^{26}$ of an isolated Zundel dimer $\left(\mathrm{H}_{5} \mathrm{O}_{2}\right)^{+}$and of an Eigen complex $\left(\mathrm{H}_{9} \mathrm{O}_{4}\right)^{+}$i.e. in the gas phase at 298 K. These reference spectra are very helpful to interpretate the existence of those distinctive species at the different states.

A common feature observed in the spectra of the excess proton in all cases is the series of maxima between 300 and $4000 \mathrm{~cm}^{-1}$. These maxima are structured into two separated ranges: one between 500 and 2000 wavenumbers and a another between 2000 and 4000 wavenumbers. Since the proton is attached to one, two or three water groups, the frequency band and maxima will account for individual (proton) and collective vibrational modes, associated to hydronium, Zundel-like or Eigen-like vibrations. As the interplate distance becomes smaller, the number of relevant maxima tend to increase. In the case of $d=0.7 \mathrm{~nm}$ the number of maxima is significantly larger than at lower $d$ and this might indicate that size effects due to the reduced space along Z-axis have been somehow captured.

Assuming that the bands located below $1000 \mathrm{~cm}^{-1}$ are typical of librational modes in water $^{79,80}$ and not characteristic of proton vibrations, we will focus our analysis on the maxima located (for the gas phase) around (see top of Fig.8): (1) 1200, 1900 and $3500 \mathrm{~cm}^{-1}$ (Zundel dimer); (2) 1450, 2750 and $3600 \mathrm{~cm}^{-1}$ (Eigen complex). First of all we should point out that our method is able to locate band maxima but it cannot reproduce heights and widths of such bands. To do so we would need to rely on a full quantum simulation (see Ref. $\left.{ }^{26}\right)$. As a general fact, locations of maxima associated to proton vibrations are in overall good qualitative agreement with experimental data. Firstly, Kobayashi et al. ${ }^{13}$ measured the frequency of the proton stretch in cubic ice around $2600 \mathrm{~cm}^{-1}$. The values of this frequency band reported in the present work oscillate between $2300 \mathrm{~cm}^{-1}$ (at $300 \mathrm{~K}$ for the case of $d=3.1 \mathrm{~nm}$ ) and $2550 \mathrm{~cm}^{-1}$ (at $500 \mathrm{~K}$ for the case of $d=0.7 \mathrm{~nm}$ ). This shift is a subtle effect due to thermodynamic and geometrical changes. The difference observed with Kobayashi's data may be attributed to the fact that the proton structure in cubic ice presents a larger degree of directionality than in the LDA states considered here. This would favor faster vibrational modes. Secondly, FTIR measurements of $\mathrm{HCl}$ and $\mathrm{NaCl}$ aqueous solutions at 
$300 \mathrm{~K}^{78}$ reported relevant maxima associated with hydrated protons around 1200, 1800 and $2900 \mathrm{~cm}^{-1}$. Further, Headrick et al. ${ }^{41}$ reported proton vibrations at $3160 \mathrm{~cm}^{-1}$ for a Zundel dimer from photoevaporation of argon in photofragmentation mass spectroscopy, which is also in reasonable good qualitative agreement with the maxima reported here. Schwartz ${ }^{81}$ also reported the finding of a frequency maximum about $2660 \mathrm{~cm}^{-1}$ for a $\mathrm{H}_{9} \mathrm{O}_{4}^{+}$cluster (Eigen complex) from infrared absorption spectra of several water clusters in gas phase. Such frequency has been attributed ${ }^{82}$ to an $\mathrm{H}$-bonded $\mathrm{H}_{3} \mathrm{O}^{+}$stretch. Finally, in a very recent work, Thämer et al. ${ }^{83}$ reported ultrafast two-dimensional infrared $2 \mathrm{D}$ spectroscopy indicating some coupling between stretching and bending vibrations of flanking waters of the Zundel dimer, located at 3200 and $1760 \mathrm{~cm}^{-1}$ respectively. Our corresponding values, as reported above, are not very far $\left(3500\right.$ and $\left.1900 \mathrm{~cm}^{-1}\right)$ and give further indication of the reliability of the present calculations.

The comparison of our findings with those from other simulation works indicates again a good overall agreement. We obtained maxima centered at 1500, 2400 and $3200 \mathrm{~cm}^{-1}$ and the results from Vuilleumier and Borgis ${ }^{48}$ (also for a flexible SPC/E model) reported stretching modes of the hydronium complex at 2000 and $2650 \mathrm{~cm}^{-1}$, whereas Voth and coworkers ${ }^{78}$ assigned modes around 1680-1880 and 3250-3400 $\mathrm{cm}^{-1}$ to vibrations of Zundel dimers and modes around 1580-1640 and 2700-2950 to vibrations of the Eigen complex. Finally, bands around 3400-3600 and 3650-3720 $\mathrm{cm}^{-1}$ were associated to a linear combination of Zundel and Eigen modes. The reported results from computed vibrational density of states by Schmitt and $\operatorname{Voth}^{49}$ for a different potential model were of 1550 and $2860 \mathrm{~cm}^{-1}$ for the two complexes.

A simple way to interpretate the spectral densities of states in Fig.8 is by means of the help of the signatures obtained from the simulation of a Zundel dimer and an Eigen complex in gas-like ambients. The agreement of the maxima with available experimental data has been previously established (see Ref. ${ }^{26}$ and references therein). The spectral band shifts will be in general a consequence of three factors: (1) the percentage of Zundel-like or Eigen-like (or monomers carrying the lone proton, such a hydronium) species existing in the system, (2) the amount of thermal energy and (3) the internal pressure of the system, directly related with confinement effects.

In the present case, the interpretation of proton vibrations through the influence of both 
confinement and temperature and the relationship with the existence of Zundel and Eigen complexes can be summarized as follows:

1. At the reference state of $300 \mathrm{~K}$, the main bands of the spectra are located around 1500 (A), 2400 (B) and $3250 \mathrm{~cm}^{-1}$ (C). No significant changes are observed as the interplate distance $d$ moves from 3.1 to $0.7 \mathrm{~nm}$. From experimental measurements of spectral signatures of water clusters ${ }^{40,40}$ it is known that the band centered at 1600 wavenumbers may be attributed to the existence of the solvated Zundel complex; the band at $2600 \mathrm{~cm}^{-1}$ is clearly due to the Eigen cation, whereas the maximum around $3200-3300 \mathrm{~cm}^{-1}$ is the signature of the $\mathrm{OH}$ stretch for the proton when shared by two waters (Zundel structure) ${ }^{83}$. At condensed phases as those analyzed here, we can expect that all frequencies suffer a red shift towards lower values, in the same fashion as it happens to hydrogen vibrations in neat non-protonated water (see for instance Ref. ${ }^{84}$ ). The effect of confinement becomes important only when the interplate distance is of (or below) $1.5 \mathrm{~nm}$. In such cases, changes in the spectral bands are observed, with a tendency of bands (A) and (B) to blue-shift by some 100-200 wavenumbers and to red-shift by 100 wavenumbers by band (C).

2. The effect of the temperature seems to be not important at the widest interplate separation $(d=3.1 \mathrm{~nm})$ but it has some influence at $d=1.5 \mathrm{~nm}$ and it definitely affects the spectral bands when $d=0.7 \mathrm{~nm}$. In such a case, LDA ice (200 K) shows a red shift of the signature band at $2400 \mathrm{~cm}^{-1}$, whereas at temperatures of 400 and 500 $\mathrm{K}$, the tendency is reversed and the shift tends to be towards higher values.

3. About Zundel-like bands, the lowest frequency one located at $1880 \mathrm{~cm}^{-1}$ in gas phase is absent in the liquid and LDA ice spectra, whereas the band at higher frequency around $3500 \mathrm{~cm}^{-1}$ appears at low frequencies in all cases. It shows a tendency to split, very marked for $d=0.7 \mathrm{~nm}$, indicating the existence of Zundel dimers or, equivalently, the breaking of Eigen groups at the strongest confinement. 


\section{CONCLUSIONS}

In the present study, a thorough analysis of the structure and dynamics of an excess proton in a wide range of temperatures ranging from LDA ices to liquid water up to sub-critical high-temperature states has been reported. We employed MD simulations together with MSEVB calculations, in order to construct a suitable Hamiltonian for the semi-classical system, formed by a quantum-like particle (the lone proton) embedded in a sea of purely classical flexible TIP3P waters. Our findings have revealed the enhancement of the local structure of the proton in LDA ices and its progressive reduction as temperature rises. At the lowest temperature $(100 \mathrm{~K})$, the environment of the proton typical of ambient conditions consisting of a mixture of Zundel and Eigen-like structures has evolved to a network of water clusters mainly formed by Zundel and Eigen complexes with enhanced directionality, as it can be deducted from our spectroscopical data. The proton in unconstrained LDA environments remains trapped in an hydronium complex for long time intervals, with a transfer time of more than $50 \mathrm{ps}$, whereas at $300 \mathrm{~K}$ the mean time for a proton transfer is of the order of 2 ps. However, as it has been indicated in some recent experimental findings ${ }^{68,85}$ PTs still occur. When the proton is confined inside the graphene channel, the PT times increase dramatically, becoming of the order of $20 \mathrm{~ns}$ when the channel is $0.7 \mathrm{~nm}$ wide. Activation energies for PT has been estimated to be in the range of $2-12 \mathrm{~kJ} / \mathrm{mol}$, in reasonable agreement with experiments ${ }^{66,67}$, which reported a value of $10 \mathrm{~kJ} / \mathrm{mol}$ for proton transfer in surface ices.

Diffusion of the proton tends to decrease when the system is cooled down, changing from $0.94 \AA^{2} / \mathrm{ps}$ at $300 \mathrm{~K}$ up to a factor 5 fold smaller at $100 \mathrm{~K}$ for the unconstrained case. Conversely, when the system is heated up, diffusion increases about a factor 3 (at 600 $\mathrm{K})$. In constrained geometries, diffusion is strongly reduced at low temperatures, becoming one order of magnitude smaller than for the bulk counterparts. When we move to high temperature environments, diffusion increases in a smaller scale reaching values comparable to those of the corresponding unconstrained states. This would suggest that confinement and temperature are factors both affecting transport properties of the proton, but playing opposite roles. In our previous study ${ }^{43}$, it was pointed out that the role of the Grotthuss mechanism should become less important at low temperatures. This was easily estimated 
using random walk arguments. In the present case we can estimate that the typical distance

$d_{\mathrm{OO}} \sim 3.5 \AA$ is trailed by a water molecule under confinement at $300 \mathrm{~K}$ (for the case of intermediate interplate distance of $1.5 \mathrm{~nm}$ ) every $93 \mathrm{ps}$, so that diffusion gets very much reduced by the effect of the constraining geometry.

\section{ACKNOWLEDGMENTS}

J.M. gratefully acknowledges financial support from Spanish MINECO for grant FIS201239443-C02-01. A.T. has been supported by Canon foundation (UK) and from Department of Physics of the Technical University of Catalonia. 


\section{References}

1. Geissler, P. L., Dellago, C., Chandler, D., Hutter, J., Parrinello, M., Science, 2001, 291, 2121-2124.

2. Trylska, J., Grochowski, P., McCammon, J. A., Protein Sci., 2004, 13, 513-528.

3. Gaigeot, M. P., Cimas, A., Seydou, M., Kim, J. Y., Lee, S., Schermann, J. P., J. Am. Chem. Soc., 2010, 132, 18067-18077.

4. Bianco, R., Hynes, J. T., Theor.Chem.Acc. 2004, 111, 182-187.

5. Müller, A., Ratajczak, H., Junge, W., Diemann, E., Electron and proton transfer in chemistry and biology; Elsevier Science: Amsterdam, 1992.

6. von Grotthus, C. J. T., Ann. Chim. (Paris) 1806, LVIII, 54-74.

7. Agmon, N., Chem. Phys. Lett. 1995, 244, 456-462.

8. Cukierman, S., Biochim. et Biophys. Acta 2006, 1757, 876-885.

9. Tuckerman, M. E., Marx, D., Klein, M. L., Parrinello, M., Science 1997, 275, 817-820.

10. Marx, D., Tuckerman, M. E., Hutter, J., Parrinello, M., Nature 1999, 397, 601-604.

11. Day, T. J. F., Schmitt, U. W., Voth, G. A., J. Am. Chem. Soc. 2000, 122, 12027-12028.

12. Kobayashi, C., Saito, S., Ohmine, I., J.Chem.Phys. 2000, 113, 9090-9100.

13. Kobayashi, C., Saito, S., Ohmine, I., J.Chem.Phys. 2001, 115, 4742-4749.

14. Walbran, S., Kornyshev, A. A., J.Chem.Phys. 2001, 114, 10039-10048.

15. Tuckerman, M. E., Marx, D., Parrinello, M., Nature 2002, 417, 925-929.

16. Bakker, H. J., Nienhuys, H.-K., Science 2002, 297, 587-590.

17. Kornyshev, A. A., Kuznetsov, A. M., Spohr, E., Ultrup, J., J. Phys. Chem. B 2003, 107, 3351-3366. 
18. Botti, A., Bruni, F., Imberti, S., Ricci, M. A., Soper, A. K., J. Chem. Phys. 2004, 121, 7840-7848.

19. Laria, D., Guàrdia, E., Martí, J., J. Am. Chem. Soc. 2004, 126, 2125-2134.

20. Asthagiri, D., Pratt, L. R., Kress, J. D., Proc. Natl. Acad. Sci. USA 2005, 102, 67046708.

21. Botti, A., Bruni, F., Ricci, M. A., Soper, A. K., J. Chem. Phys. 2006, 125, 014508.

22. Voth, G. A., Acc. Chem. Res. 2006, 39, 143-150.

23. Marx, D. Chem. Phys. Chem. 2006, 7, 1848-1870.

24. Swanson, J. M. J., Maupin, C. M., Chen, H., Petersen, M. K., Xu, J., Wu, Y., Voth, G. A., J. Phys. Chem. B 2007, 111, 4300-4314.

25. Lee, S. H., Rasaiah, J. C., J. Chem. Phys. 2011, 135, 124505.

26. Tahat, A., Martí, J., Phys. Rev. E 2014, 89, 052130.

27. Chernyshev, A., Cukierman, S., Biophys. J. 2002, 82, 182-192.

28. Le Caër, S., Palmer, D. J., Lima, M., Renault, J. P., Vigneron, G., Righini, R., Pommeret, S., J. Am. Chem. Soc. 2009, 129, 11720-11729.

29. Wang, C., Waje, M., Wang, X., Tang, J. M., Haddon, R. C., Yan, Y., NanoLett. 2004, $4,345-348$.

30. Spry, D. B., Goun, A., Glusac, K., Moilanen, D. E., Fayer, M. D:, J. Am. Chem. Soc. 2007, 129, 8122-8130.

31. Hynes, J. T., Klinman, J. P., Limbach, H.-H., Schowen, R. L., Eds.; Hydrogen-transfer reactions; John Wiley and sons: New York, 2006.

32. Soper, A. K., Mol. Phys. 2008, 106, 2053-2076.

33. Vega, C., Abascal, J. L. F., Conde, M. M., Aragones, J. L., Faraday Discuss. 2009, 141, 251-276. 
34. Cao, Z., Peng, Y., Yan, T., Li, S., Li, A., Voth, G. A., J. Am. Chem. Soc. 2010, 132, 11395-11397.

35. Hummer, G., Rasaiah, J. C., Noworyta, J. P., Nature, 2001, 414, 188-190.

36. Wolf, M. G., Groenhof, G., J. Comput. Chem. 2014, 35, 657-671.

37. Bankura, A., Chandra, A., J. Phys. Chem. B, 2012, 116, 9744-9757.

38. Clark, J. K., Paddison, S. J., Phys. Chem. Chem. Phys. 2014, 16, 17756-17769.

39. Bankura, A., Chandra, A., J. Chem. Phys. 2015, 142, 044701.

40. Hammer, N. I., Diken, E. G., Roscioli, J. R., Johnson, M. A., Myshakin, E. M., Jordan, K. D., McCoy, A. B., Huang, X., Bowman, J. M., Carter, S., J. Chem. Phys. 2005, 122, 244301.

41. Headrick, J. M., Diken, E. G., Walters, R. S., Hammer, N. I., Christie, R. A., Cui, J., Myshakin, E. M., Duncan, M.A., Johnson, M. A., Jordan, K. D., Science, 2005, 308, 1765-1769.

42. Cheng, T. C., Bandyopadhyay, B., Mosley, J. D., Duncan, M. A., J. Am. Chem. Soc. 2012, 134, 13046-13055.

43. Tahat, A., Martí, J., Phys. Rev. E 2015, 92, 032402.

44. Warshel, A., Computer Modeling of Chemical Reactions in Enzymes and Solutions; Wiley: New York, 1980.

45. Aqvist, J., Warshel, A., Chem. Rev. 1993, 93, 2523-2544.

46. Lobaugh, J., Voth, G. A., J. Chem. Phys. 1996, 104, 2056-2069.

47. Sagnella, D. E., Tuckerman, M. E., J. Chem. Phys. 1998, 108, 2073-2083.

48. Vuilleumier, R., Borgis, D., J. Chem. Phys. 1999, 111, 4251-4266.

49. Schmitt, U. W., Voth, G. A., J. Chem. Phys. 1999, 111, 9361-9381. 
50. Day, T. J. F., Soudackov, A. V., Cuma, M., Schmitt, U. W., Voth, G. A., J. Chem. Phys. 2002, 117, 5839-5849.

51. Tahat, A., Martí, J., Khwaldeh, A., Tahat, K., Chin. Phys. B 2014, 23, 046101.

52. Dang, L. X., Pettitt, B. M., J. Phys. Chem. 1987, 91, 3349-3354.

53. Gordillo, M. C., Martí, J. Chem. Phys. Lett. 329, 341-345 (2000).

54. Spohr, E., J. Chem. Phys. 1997, 107, 6342-6348.

55. Frenkel, D., Smit, B., Understanding Molecular Simulation; Academic Press: San Diego, 2002.

56. Hub, J. S., de Groot, B. L., Grubmüller, H., Groenhof, G. J. Chem. Theor. Comput. 2014, 10, 381-390.

57. Zundel, G., Metzger, H., Z. Phys. Chem. 1968, 58, 225-245.

58. Eigen, M., de Maeyer, L., Proc. Royal Soc. (London) 1958, 247, 505-533.

59. Tuckerman, M., Laasonen, K., Sprik, M., Parrinello, M., J. Chem. Phys. 1995, 103, $150-161$.

60. Tuckerman, M., Laasonen, K., Sprik, M., Parrinello, M., J. Phys. Chem. 1995, 99, $5749-5752$.

61. Meiboom, S., J. Chem. Phys. 1961, 34, 375-388.

62. Hertz, H. G., Z. Phys. Chem. 1983, 135, 89-105.

63. Pfeifer, R., Hertz, H. G., Ber. Bunsen-Ges. Phys. Chem. 1990, 94, 1349-1353.

64. Semino, R., Laria, D., J. Chem. Phys. 2012, 136, 194503.

65. Chandler, D., Introduction to Modern Statistical Mechanics; Oxford University Press: New York, 1987.

66. Moon, E-S., Yoon, J., Kang, H., J. Chem. Phys. 2010, 133, 044709. 
67. Park, S-C., Moon, E-S., Kang, H., Phys. Chem. Chem. Phys. 2010, 12, 12000-12011.

68. Kim, J. H., Kim, Y-K., Kang, H., J. Chem. Phys. 2009, 131, 044705.

69. Luz, Z., Meiboom, S., J. Am. Chem. Soc. 1964, 86, 4768-4769.

70. Yeh, I-C., Hummer, G., J. Phys. Chem. B 2004, 108, 15873-15879.

71. Liu, P., Harder, E., Berne, B. J., J. Phys. Chem. B 2004, 108, 6595-6602.

72. Robinson, R. A., Stokes, R. H., Electrolyte Solutions; Butterworths: London, 1959.

73. Krynicki, K., Green, C. D., Sawyer, D. W., Faraday Discus. Chem. Soc. 1978, 66, 199-208.

74. von Hippel, A., IEEE Transact. Elec. Insul. 1988, 23, 825-840.

75. Petersen, M. K., Iyengar, S. S., Day, T. J. F., Voth, G. A., J. Phys. Chem. B 2004, 108, 14804-14806.

76. Martí, J., Sala, J., Gordillo, M. C., Guàrdia, E., J., Phys. Rev. E 2009, 79, 031606.

77. McQuarrie, D. A., Statistical Mechanics; University Science Books: Sausalito, California, 2000.

78. Kim, J., Schmitt, U. W., Gruetzmacher, J. A., Voth, G. A., Scherer, N. E., J. Chem. Phys. 2002, 116, 737-746.

79. Martí, J., Padró, J. A., Guàrdia, E., Mol. Phys. 1995, 86, 263-271.

80. Martí, J., Padró, J. A., Guàrdia, E., J. Chem. Phys. 1996, 105, 639-649.

81. Schwarz, H. A., J. Chem. Phys. 1977, 67, 5525-5534.

82. Okumura, M., Yeh, L. I., Myers, J. D., Lee, Y. T., J. Phys. Chem. 1990, 94, 3416-3427.

83. Thämer, M., De Marco, L., Ramasesha, K., MAndal, A., Tokmakoff, A., Science 2015, $350,78-82$. 
84. Eisenberg, D., Kauzmann, W., The structure and properties of water; Oxford University Press: London, 1969.

85. Lee, C-W., Lee, P-R., Kim, Y-K. Kang, H. J. Chem. Phys. 2007, 127, 084701. 
Figure 1: Pivot oxygen-solvent oxygen density fields (left side) and pivot oxygen-solvent hydrogen desnity fields (right side) at different thermodynamic states. Interplate separations: $d=3.1 \mathrm{~nm}$ (top figures); $d=1.5 \mathrm{~nm}$ (middle figures) and $d=0.7 \mathrm{~nm}$ (bottom figures).

Figure 2: Snapshots of local configurations around the pivot water confined inside the graphene slab $1.5 \mathrm{~nm}$ wide at different thermodynamic states (left to right): $\mathrm{T}=100$, 300 and $500 \mathrm{~K}$. Only water molecules having largest coefficients $c_{i}$ (usually 15-25 molecules) have been shown.

Figure 3: Time evolution of pivot-oxygen labelling in different aqueous environments, from 100 to $600 \mathrm{~K}$ and for all interplate separations.

Figure 4: Logarithm of the population relaxations at different thermodynamical states and interplate separations.

Figure 5: Proton transfer rates as a function of temperature and interplate separation. The straight lines represent the best overall linear fits: bulk unconstrained systems (black line); $d=3.1 \mathrm{~nm}$ (red line); $d=1.5 \mathrm{~nm}$ (green line) and $d=0.7 \mathrm{~nm}$ (blue line). Dashed lines are best linear fits for temperatures between 200 and $600 \mathrm{~K}$.

Figure 6: Mean square displacements of proton species at different interplate separations (left to right): $d=3.1,1.5$ and $0.7 \mathrm{~nm}$ as a function of temperature.

Figure 7: Diffusion coefficients of the excess proton as a function of inverse temperature for variable interplate distances. Straight lines represent the best overall linear fits, whereas dashed lines account for fits between 200 and $600 \mathrm{~K}$.

Figure 8: Vibrational densities of states $S_{p}(\omega)$ (in arbitrary units): $d=3.1,1.5$ and $0.7 \mathrm{~nm}$. Proton in Zundel and Eigen complexes in gas phase at $298 \mathrm{~K}$ have been also included. 

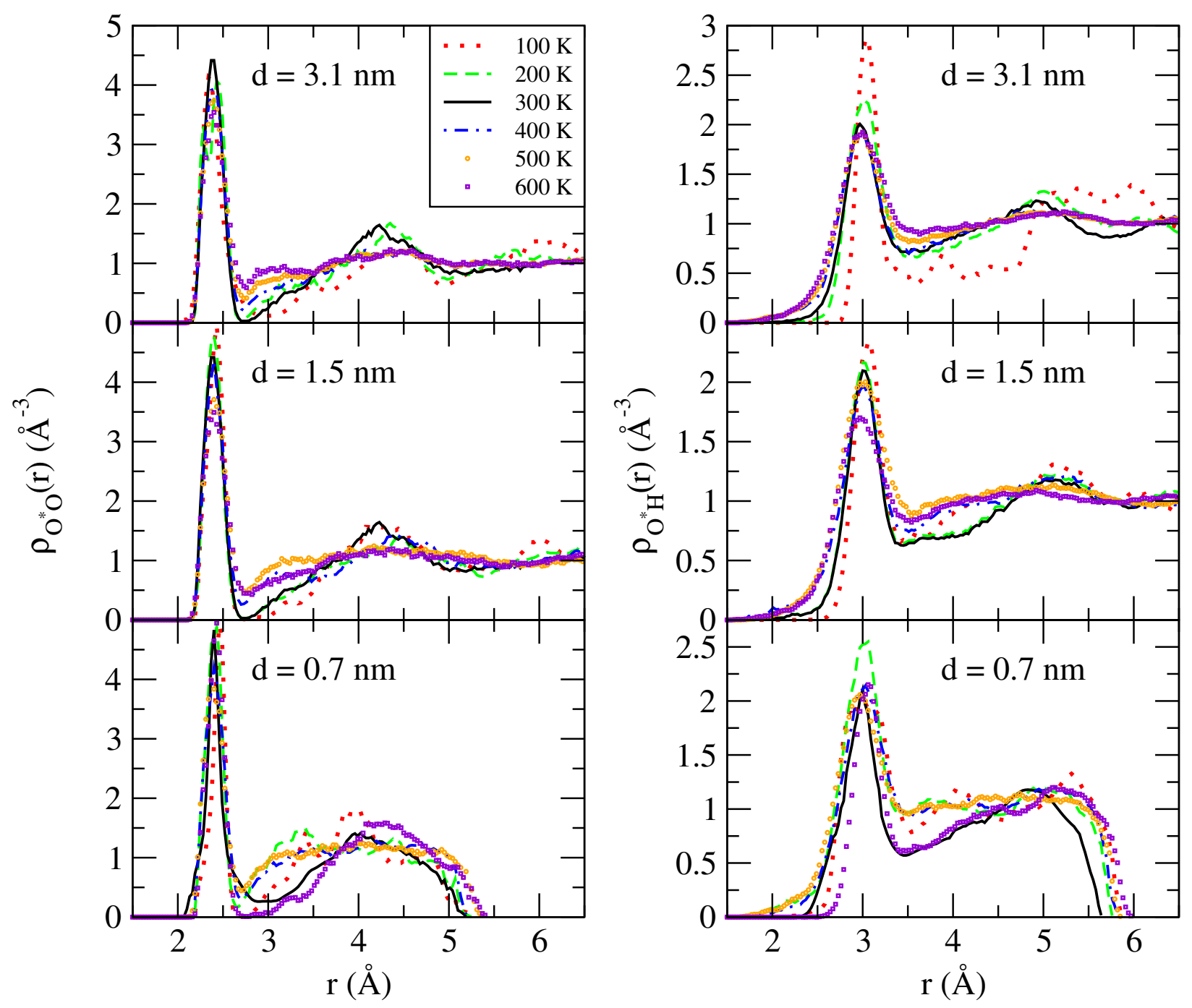

Figure 1

Tahat, Marti

J. Comput. Chem. 

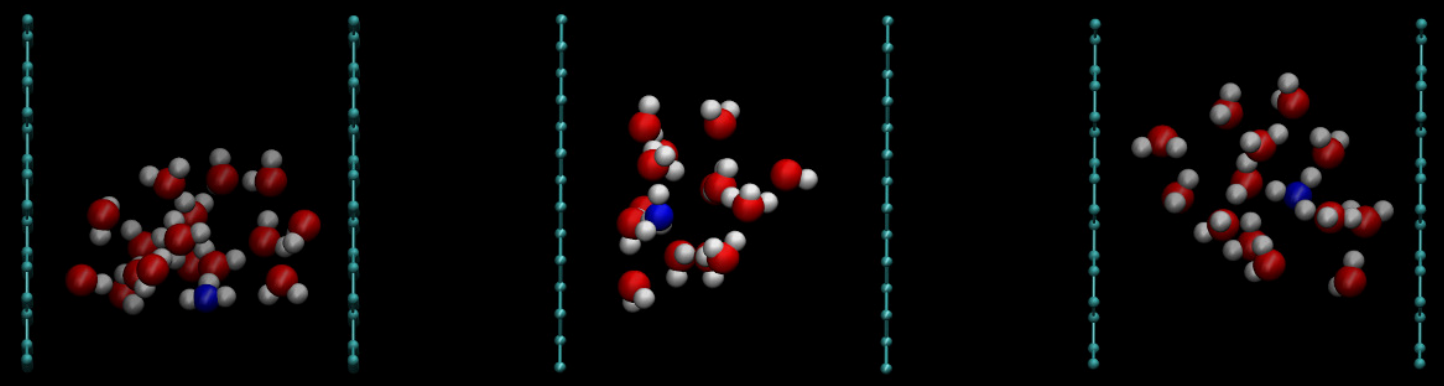

Figure 2

Tahat, Marti

J. Comput. Chem. 


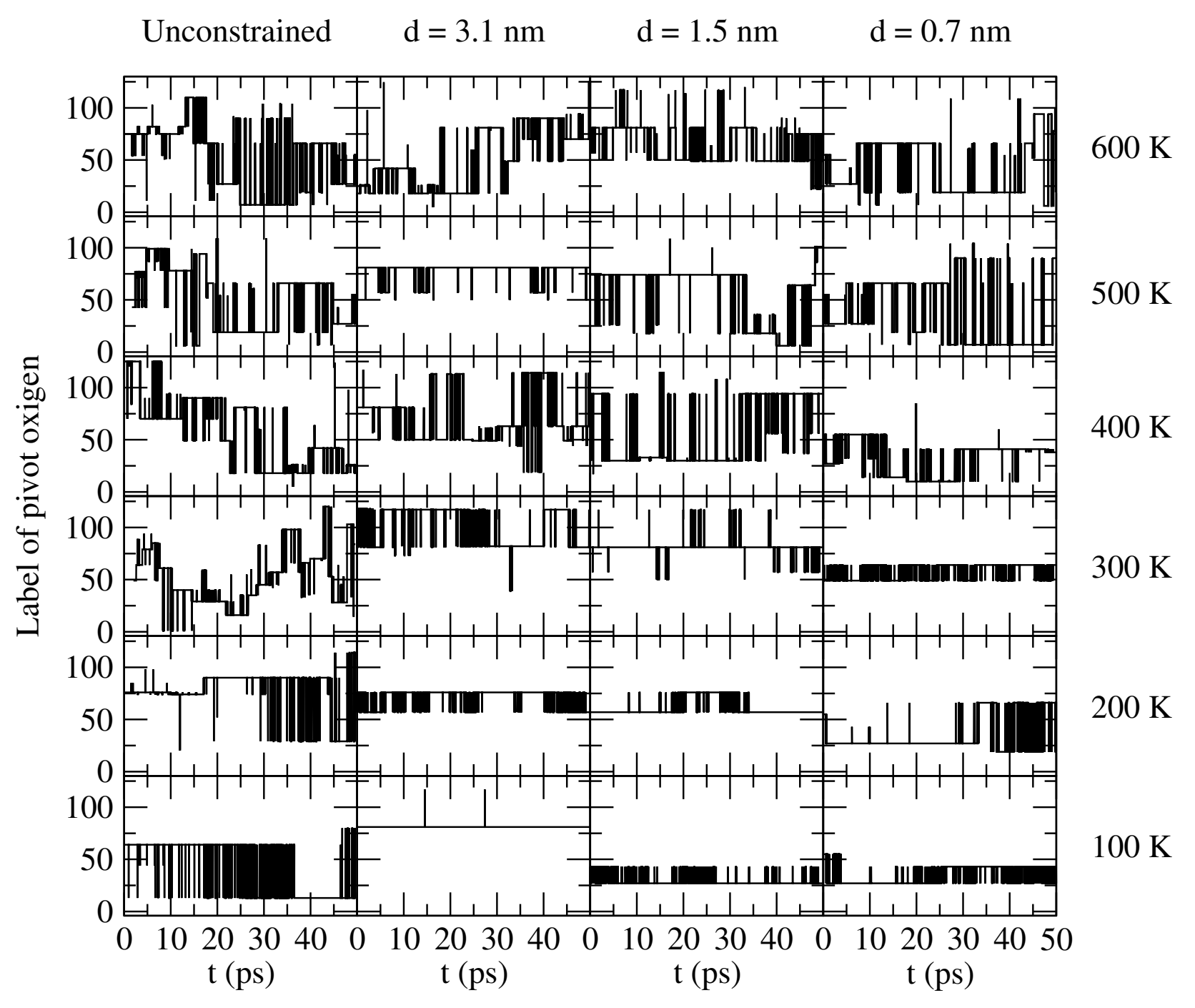

Figure 3

Tahat, Marti

J. Comput. Chem. 


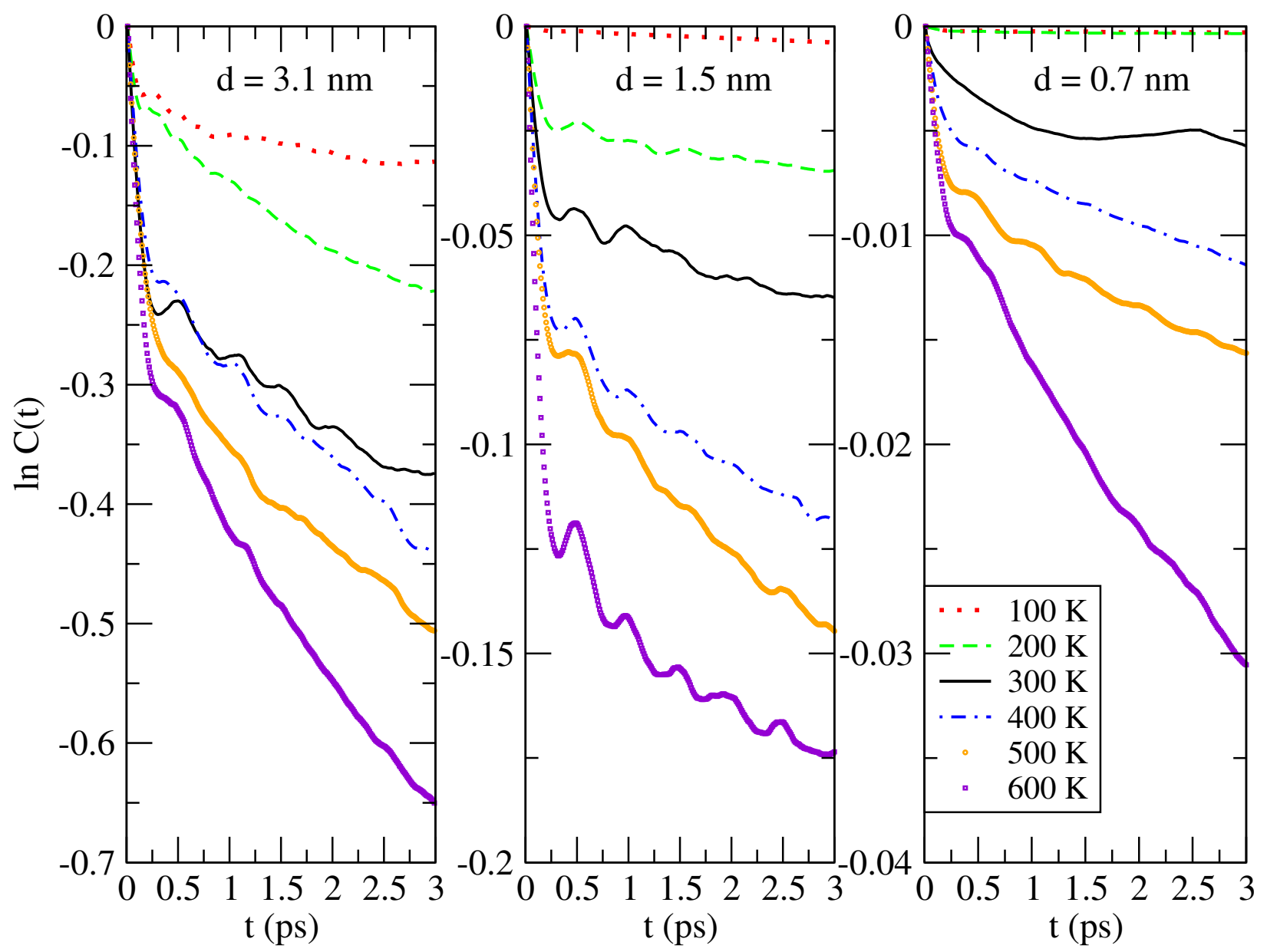

Figure 4

Tahat, Marti

J. Comput. Chem. 


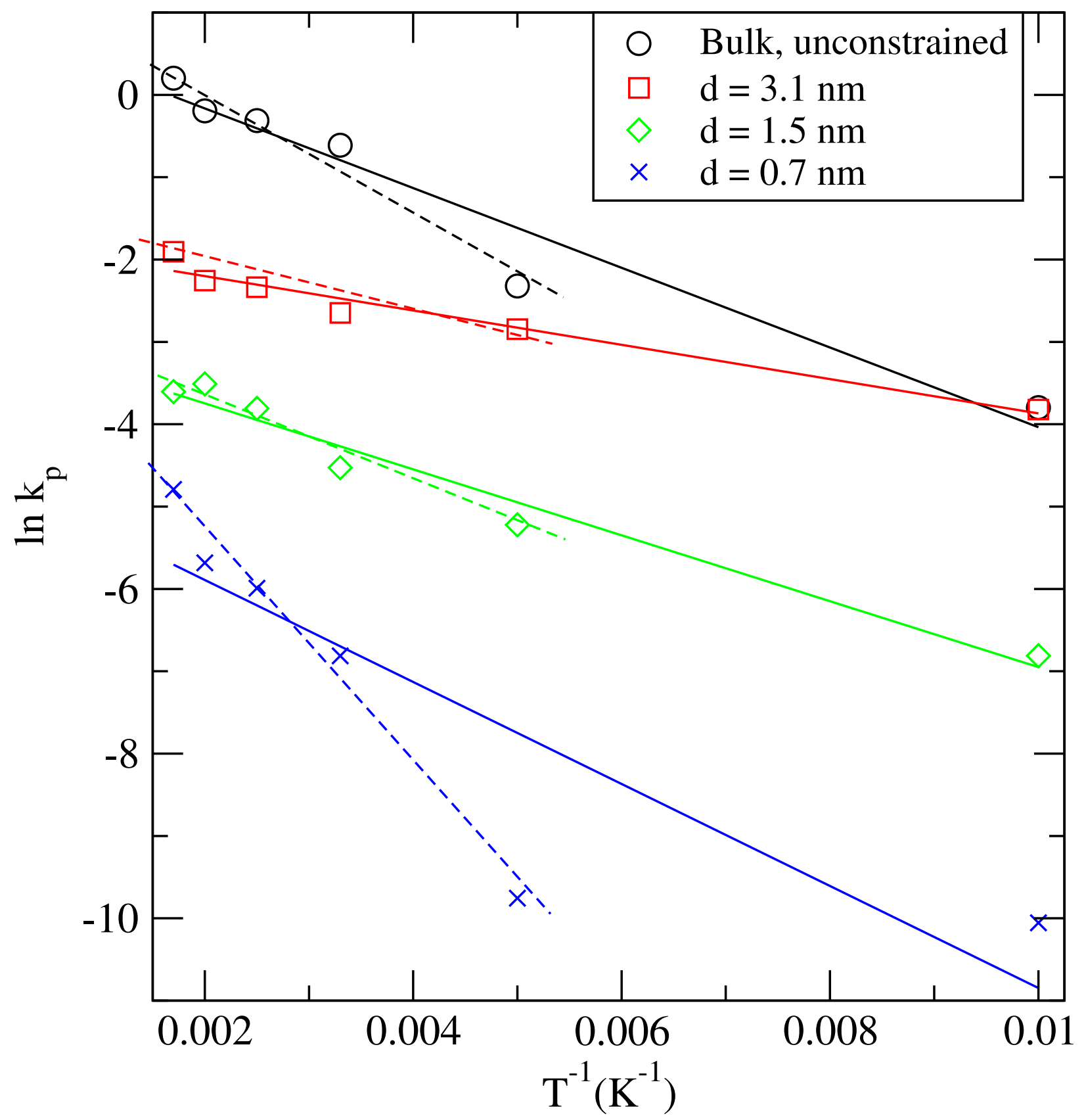

Figure 5

Tahat, Marti

J. Comput. Chem. 


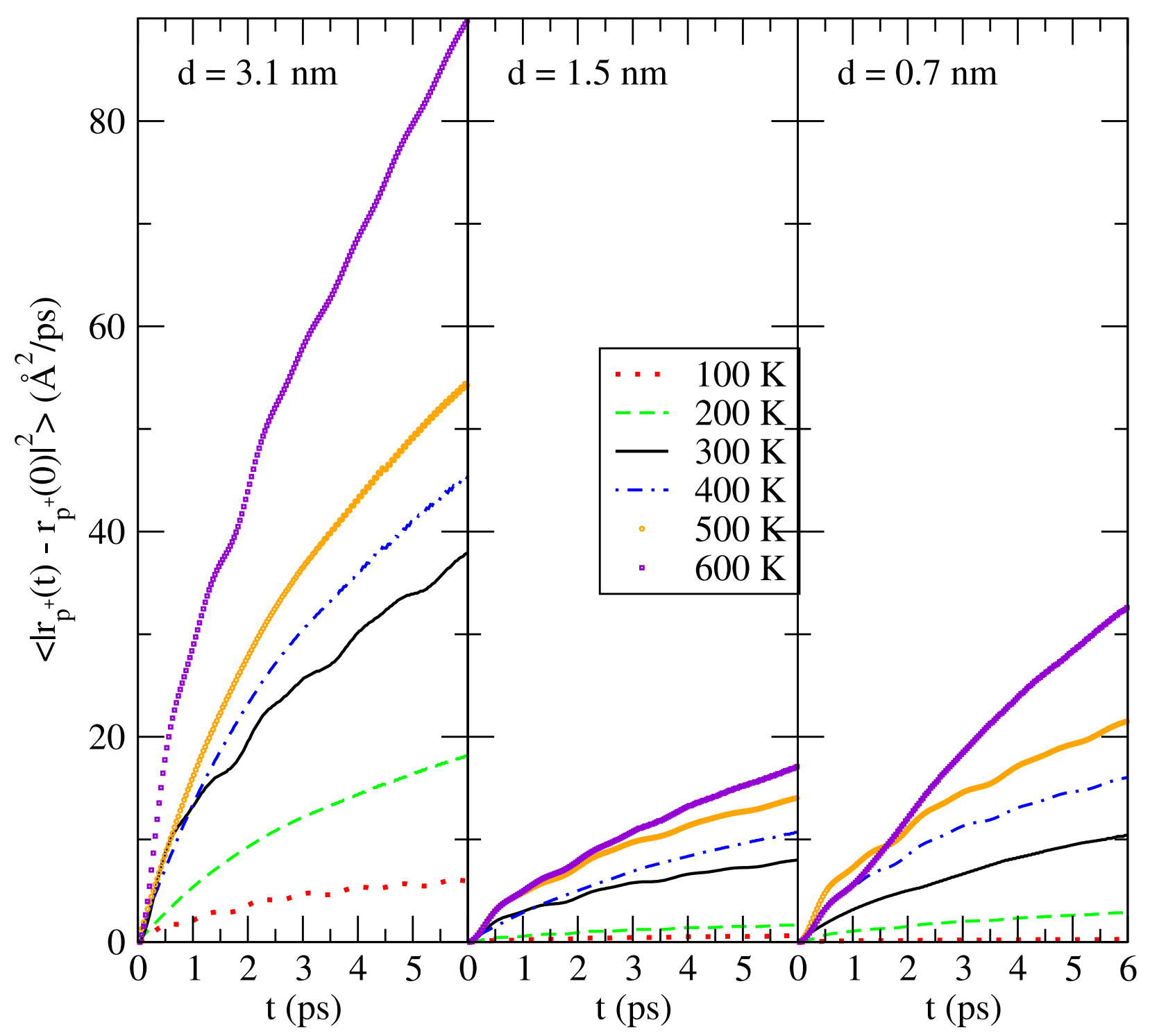

Figure 6

Tahat, Marti

J. Comput. Chem. 


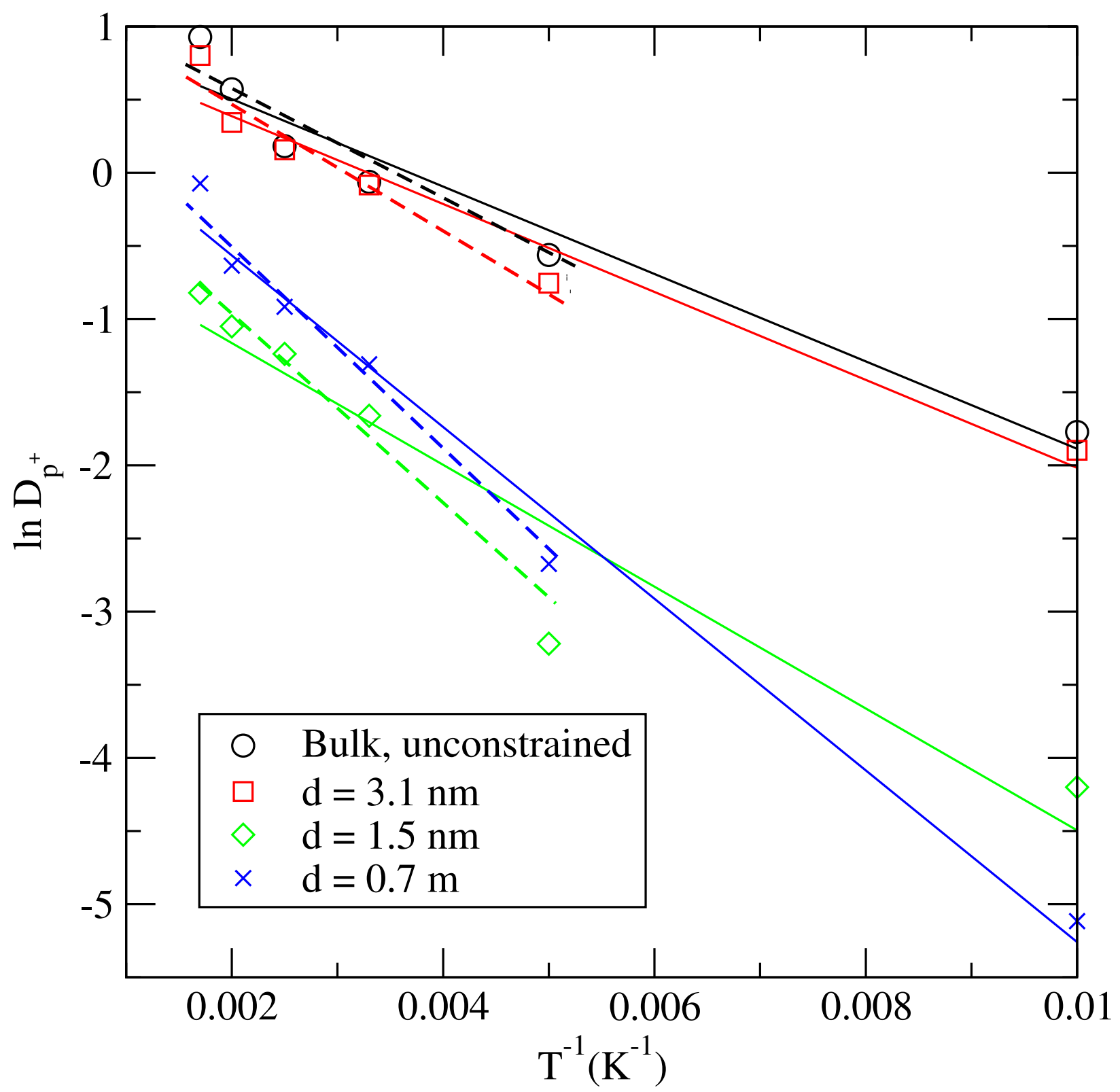

Figure 7

Tahat, Marti

J. Comput. Chem. 


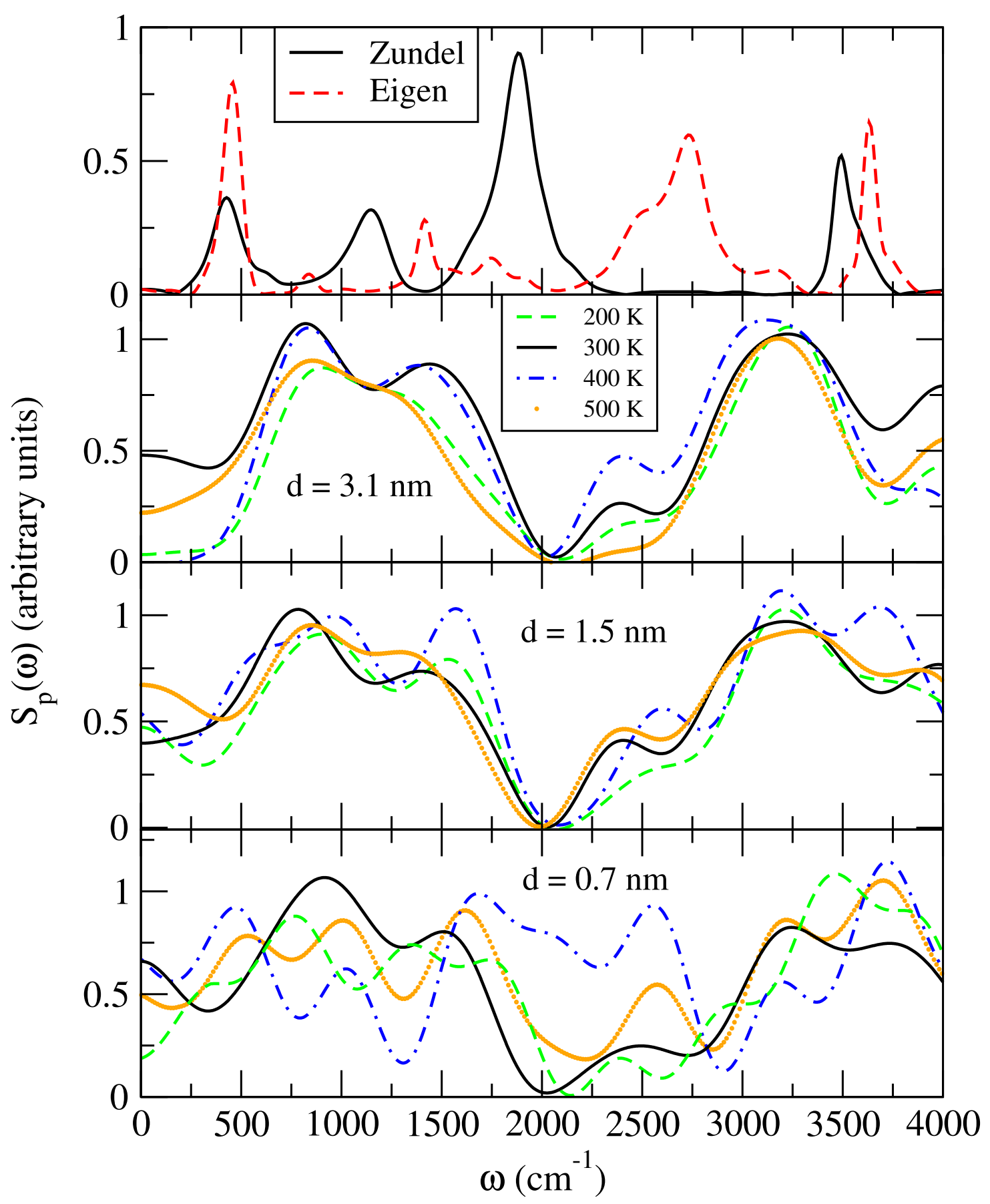

Figure 8

Tahat, Marti

J. Comput. Chem. 


\begin{tabular}{|c|c|c|c|c|c|c|c|}
\hline \multirow[t]{2}{*}{ d } & & \multicolumn{6}{|c|}{$\mathbf{T}$} \\
\hline & & 100 & 200 & 300 & 400 & 500 & 600 \\
\hline & $k_{p}$ & $0.022(0.012)$ & $0.098(0.036)$ & $0.543(0.230)$ & $0.732(0.076)$ & $0.823(0.056)$ & $1.225(0.080)$ \\
\hline \multirow[t]{3}{*}{ Unconstrained } & $\tau_{r s d}$ & $44.6(0.3)$ & $10.2(0.1)$ & $1.80(0.01)$ & $1.41(0.06)$ & $1.22(0.05)$ & $0.81(0.05)$ \\
\hline & $D_{p}+$ & $0.17(0.11)$ & $0.57(0.13)$ & $0.94(0.10)$ & $1.20(0.17)$ & $1.77(0.31)$ & $2.53(0.11)$ \\
\hline & $k_{p}$ & $0.022(0.010)$ & $0.058(0.030)$ & $0.071(0.03)$ & $0.097(0.010)$ & $0.105(0.007)$ & $0.149(0.009)$ \\
\hline \multirow[t]{3}{*}{3.1} & $\tau_{r s d}$ & $45.7(0.3)$ & $17.2(0.1)$ & $14.1(0.1)$ & $10.3(0.4)$ & $9.5(0.4)$ & $6.7(0.5)$ \\
\hline & $D_{p}+$ & $0.15(0.08)$ & $0.47(0.06)$ & $0.92(0.11)$ & $1.17(0.16)$ & $1.41(0.24)$ & $2.23(0.21)$ \\
\hline & $k_{p}$ & $0.0011(0.0005)$ & $0.0054(0.0002)$ & $0.011(0.004)$ & $0.022(0.002)$ & $0.030(0.002)$ & $0.027(0.001)$ \\
\hline \multirow[t]{3}{*}{1.5} & $\tau_{r s d}$ & $909(6)$ & $185(1)$ & $92.6(0.3)$ & $45.0(1.6)$ & $33.4(1.3)$ & $36.8(2.7)$ \\
\hline & $D_{p}+$ & $0.015(0.010)$ & $0.040(0.007)$ & $0.19(0.02)$ & $0.29(0.04)$ & $0.35(0.06)$ & $0.44(0.04)$ \\
\hline & $k_{p}$ & $4(2) \times 10^{-5}$ & $6(4) \times 10^{-5}$ & $0.0011(0.0004)$ & $0.0025(0.0003)$ & $0.0034(0.0002)$ & $0.0083(0.0005)$ \\
\hline \multirow[t]{2}{*}{0.7} & $\tau_{r s d}$ & $23256(117)$ & $17241(96)$ & $909(28)$ & $400(17)$ & $294(11)$ & $121(9)$ \\
\hline & $D_{p}+$ & $0.006(0.005)$ & $0.069(0.011)$ & $0.27(0.03)$ & $0.40(0.05)$ & $0.53(0.03)$ & $0.93(0.04)$ \\
\hline
\end{tabular}

Table 1: Dynamical parameters for the aqueous $\mathrm{H}^{+}$at different thermodynamic states: proton transfer rates $k_{p}$ in $\mathrm{ps}^{-1}$, residence time of proton $\tau_{r s d}$ in ps and diffusion coefficient of the lone proton $D_{p^{+}}$in $\AA^{2} / \mathrm{ps}$. d is the interplate distance in $\mathrm{nm}$ and $\mathrm{T}$ is the temperature in $\mathrm{K}$. The errors in all values are indicated in parenthesis. They were obtained from two independent simulations of length 0.125 ns each.

\begin{tabular}{|c|c|c|c|c|}
\hline $\mathbf{d}$ & \multicolumn{4}{|c|}{$E_{k}$} \\
\hline \hline & PT (full) & PT (5-point) & D (full) & D (5-point) \\
\hline Unconstrained & $4.02(0.03)$ & $6.03(0.11)$ & $2.49(0.05)$ & $3.46(0.04)$ \\
\hline $\mathbf{3 . 1}$ & $1.73(0.04)$ & $2.13(0.05)$ & $2.50(0.05)$ & $3.52(0.06)$ \\
\hline $\mathbf{1 . 5}$ & $3.33(0.05)$ & $4.49(0.06)$ & $3.46(0.03)$ & $5.96(0.07)$ \\
\hline $\mathbf{0 . 7}$ & $5.15(0.08)$ & $11.85(0.21)$ & $4.88(0.06)$ & $6.09(0.08)$ \\
\hline \hline
\end{tabular}

Table 2: Activation energies $\left(E_{k}\right.$, in $\left.\mathrm{kJ} / \mathrm{mol}\right)$ for the aqueous $\mathrm{H}^{+}$at different interplate separations d (in $\mathrm{nm}$ ): PT stands for proton transfer activation energy and D for proton diffusion activation energy. "Full" corresponds to the fit of all inverse temperatures in Figs.5 and 7 (continuous lines) whereas "5-point" stands for the fit for inverse temperatures between 0.0017 and 0.005 in Figs. 5 and 7 (dashed lines). The errors in all values are indicated in parenthesis and were obtained from two independent calculations. 